\title{
Numerical simulation of spin transport in systems with complex geometry
}

\author{
Timofey Andrianov ${ }^{1, *}$, and Anatoly Vedyayev ${ }^{1,2}$ \\ ${ }^{1}$ M. V. Lomonosov Moscow State University, Faculty of Physics, Leninskie Gory 1, 1199991 Moscow, Russia \\ ${ }^{2}$ SPINTEC, UMR CEA/CNRS/UJF-Grenoble1/Grenoble-INP, INAC, 38054 Grenoble, France
}

\begin{abstract}
The spin diffusion and charge equations in Levy-Fert and Waintal models were numerically solved, using finite element method in complex non-collinear geometry with strongly inhomogeneous current flow. As an illustration, spin-dependent transport through a magnetic pillar and nonmagnetic spacer separating two magnetic layers was investigated. It is shown, that the structure with number of pillars gives a higher value of Giant Magnetoresistance (GMR) effect rather than a structure with one pillar of equivalent diameter. The inhomogeneity of spin currents, which has one of the strongest impacts on GMR effect value leads to the occurrence of spin-current vortices. Introduction of $l_{T}$ and $l_{L}$ lengths in Waintal model gives a better description of angular dependence of GMR effect rather than Levy-Fert model.
\end{abstract}

\section{Introduction}

Discovery of the effect of giant magnetoresistance (GMR) was a first step which allowed spintronics to become a very promising field of studies $[1,2]$. The scrutinized investigation of this effect allowed creating a new revolutionary type of reading heads for hard disc drives, which gave a huge leap for computer technologies development. The GMR effect was explained in terms of spin-dependent scattering phenomena, which takes place at the interfaces and in the adjacent magnetic layers. Later a few theoretical models of GMR effect were offered for geometries current-in-plane (CIP) [3,4] and current-perpendicularplane (CPP) [5-8]. One of the ground theories introduces the concept of spin accumulation and spin diffusion length as key parameters to describe the diffusive transport in CPP metallic multilayers, which were generalized for both collinear and non-collinear cases. Recently another model was proposed by Waintal et al [9] which generalizes Valet-Fert theory, involving two new coherence and Larmor lengths. However, all these theoretical models were applied for simple geometries with homogeneous current flow so far. However, many spintronic devices involve inhomogeneous current flows. In our paper, we numerically investigate the structures with geometries that cause non-uniform current flows, using Levy-Fert [6] and Waintal [9] transport models for comparison. We show that inhomogeneity of current flows, appearing in GMR structures leads to creation of vortices in distribution of spin currents and compare angular dependence of GMR for Levy-Fert and Waintal models.

\section{Method}

Here we investigate and compare two type of structures. The first one consists of two 5-nm thick ferromagnetic layers connected with paramagnetic spacer of variable size sandwiched between two 100-nm thick paramagnetic electrodes (Fig. 1).
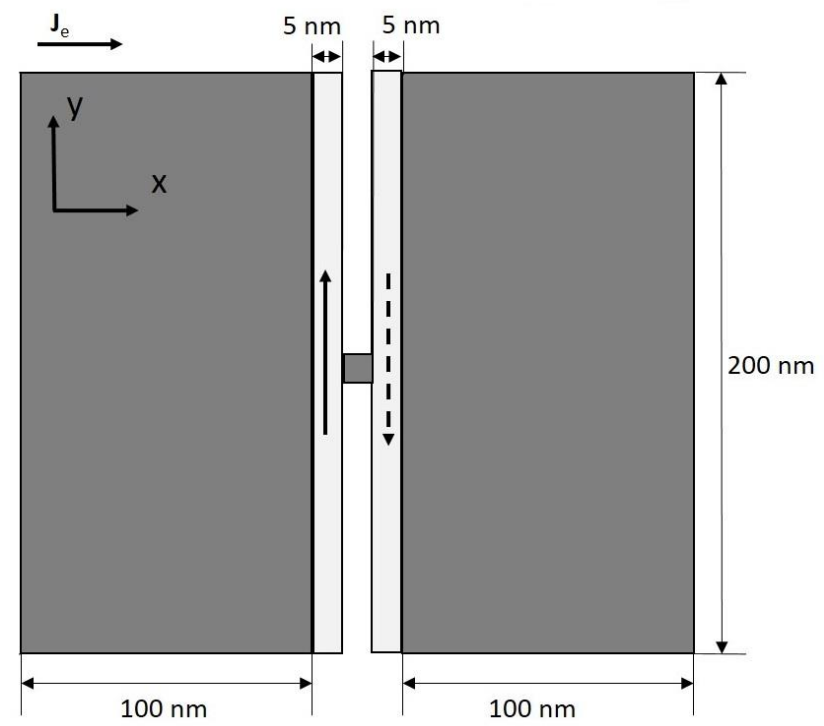

Fig.1. Structure with metallic spacer used for the finite element calculation of CPP spin transport through a nanoconstricted spin valve. In case of 2D model, all quantities are calculated per $1 \mathrm{~nm}$ depth.

The second structure consists of so-called magnetic pillars sandwiched between two 100-nm thick paramagnetic electrodes (Fig. 2).

\footnotetext{
* Timofey Andrianov: timofey.andrianov@gmail.com
} 


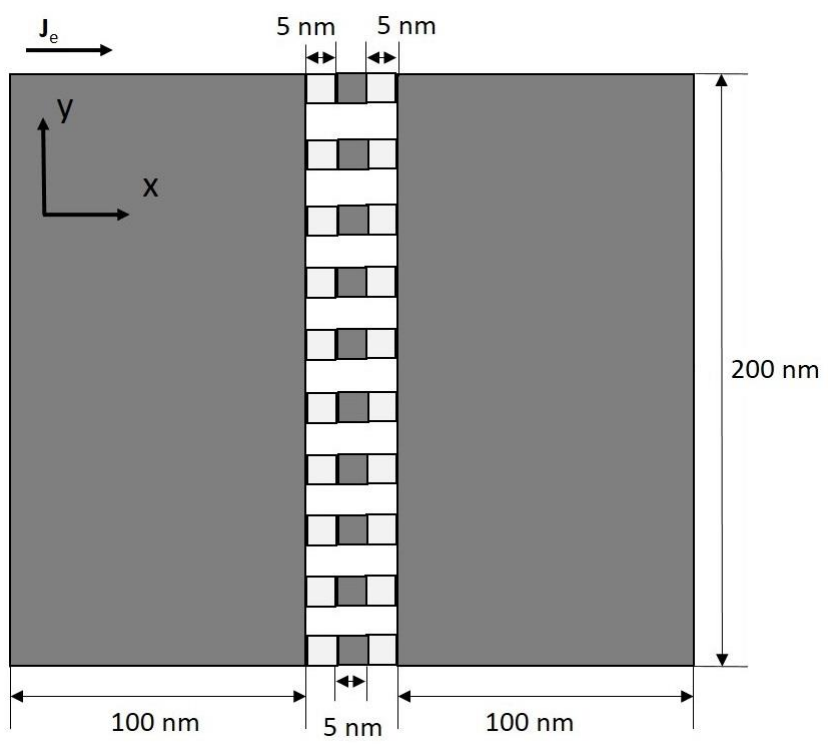

Fig.2. Structure with magnetic pillar used for the finite element calculation of CPP spin transport through a nanoconstricted spin valve. In case of 2D model, all quantities are calculated per $1 \mathrm{~nm}$ depth.

The pillar is a thin tube corresponding to a simple spin valve with number of repeating ferromagnetic and paramagnetic layers. In both structures, one of the ferromagnetic layers has a fixed magnetization directed perpendicular to current flow. Magnetization in the other layer rotates from parallel to antiparallel state relatively to the first one.

In our numerical simulation, we use and compare two models which describe spin transport in the structures. The diffusive spin transport equations proposed by Levy-Fert extended on non-collinear geometry look as follows [6]:

$$
\begin{gathered}
\mathbf{J}_{\mathbf{e}}=-\sigma \nabla \varphi-\beta \frac{\sigma}{v}(\mathbf{M}, \nabla \mathbf{m})(1) \\
\mathbf{J}_{\mathbf{m}}=-\sigma \beta(\mathbf{M}, \nabla \varphi)-\frac{\sigma}{v} \nabla \mathbf{m}(2) \\
\operatorname{div}_{\mathbf{e}}=0(3) \\
\operatorname{div} \mathbf{J}_{\mathbf{m}}=-\frac{\sigma}{v l_{s f}^{2}} \mathbf{m}-\frac{\sigma}{v l_{\mathrm{J}}^{2}}[\mathbf{M}, \mathbf{m}](4)
\end{gathered}
$$

Where $\varphi$ - electric potential, $\sigma$ - conductivity, $\beta-$ parameter of spin asymmetry of conductivity, $\mathbf{M}-$ unit vector of magnetization; $\mathbf{m}$ - spin accumulation vector, which has 3 components in spin dimension, $l_{s f}-$ spin diffusion length, $l_{J}$ - precession length, $v-$ density of states at Fermi level. Unless electric current, spin current is not conserved and can vary either because of spin relaxation or because of the local spin-transfer torque, which induces a precession of the spin accumulation around the local magnetization due to $s-d$ exchange interaction.
Spin transport equations presented by Waintal et al derived directly from Valet-Fert [8] theory and introduces two new characteristic lengths: the transverse spin coherence length $l_{T}$ and the (Larmor) spin precession length $l_{\mathrm{L}}$. The first length is responsible for decaying and the second one for precession. Introduction of these two lengths give a better description of the angular dependence of GMR in studied structures.

Here we take into account only bulk spin-dependent scattering, which would not change qualitative description of the phenomena presented in paper.

The numerical simulation was provided with finite element method on the basis of Comsol Multiphysics ${ }^{\circledR}$ software. For all simulations, we used the following values of the key parameters: $\sigma^{F}=0.001(\Omega \cdot \mathrm{nm})^{-1}$; $\sigma^{P}=0.01(\Omega \cdot \mathrm{nm})^{-1} ; \nu=0.1(\mathrm{eV})^{-1} ; l_{s f}{ }^{P}=100 \mathrm{~nm} ; \beta=0.7$; $J_{e}=10^{8} \mathrm{~A} / \mathrm{cm}^{2} ; l_{J}=1 \mathrm{~nm}$, where indexes $F$ and $P$ refer to ferromagnetic and paramagnetic layers accordingly.

\section{Results}

First, we compared two different geometries. The first one has long paramagnetic electrodes with long and thin ferromagnetic layers connected with a thin paramagnetic spacer. The second has two paramagnetic electrodes connected with one or several thin pillars in form of F/P/F. In the Fig. 3 dependence of GMR on diameter of pillar and spacer is shown.

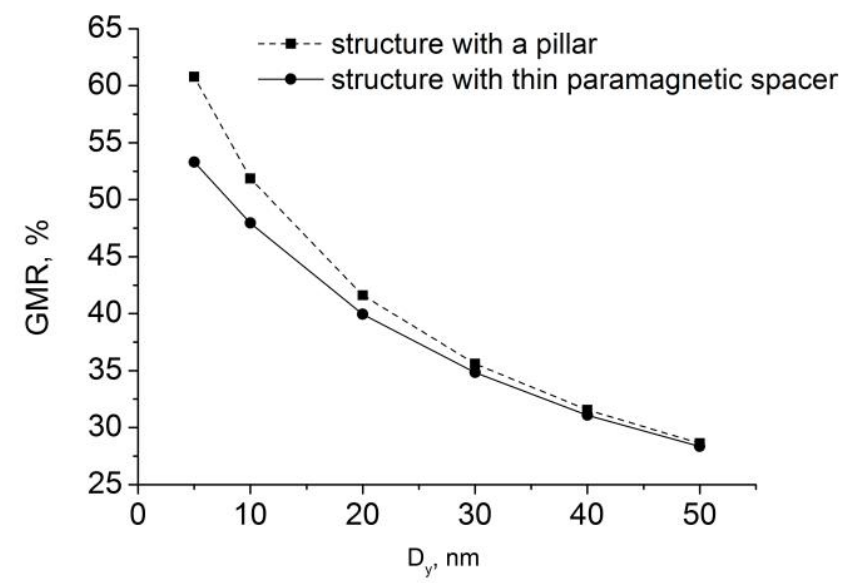

Fig.3. Dependence of GMR from diameters of pillar and spacer. $l_{s f} F=10 \mathrm{~nm}$.

The result of numerical simulation showed that in structure with $\mathrm{F} / \mathrm{P} / \mathrm{F}$ pillar of small diameter the value of GMR effect is higher than in structure with paramagnetic spacer of the same diameter.

As the next step, we investigated the system where electrodes connected with different number of $\mathrm{F} / \mathrm{P} / \mathrm{F}$ pillars. For this geometry GMR effect was calculated and compared with dependence of GMR for the geometry with one pillar of equivalent diameter (Fig.4). Naturally, due to decreasing of total resistance of system with pillars the value of GMR effect decreases with the increasing of diameter or number of pillars. Fig.4 shows that geometry with number of pillars gives significantly higher GMR effect rather than geometry with one pillar of equivalent diameter. It means that one of the most 
important impacts on GMR effect makes a non-uniform distribution of spin currents in the investigated system [10].

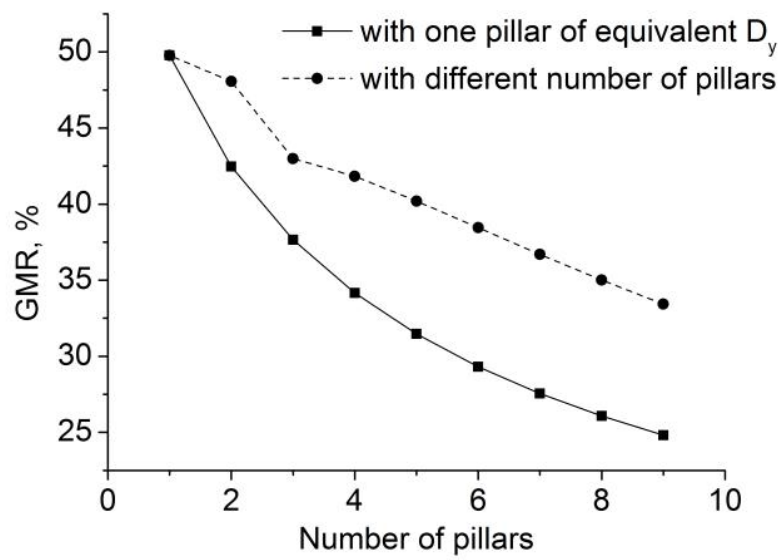

Fig.4. Dependence of GMR from number of pillars and one pillar with equivalent diameter. $l_{s f}^{F}=5 \mathrm{~nm}$.

To demonstrate non-uniform behavior of spin current in the structure we plot spin current streamlines. One can notice that in some regions of the structure the streamlines started to curl forming spin current vortices, which can be considered as inhomogeneity of spin current distribution (Fig. 5).

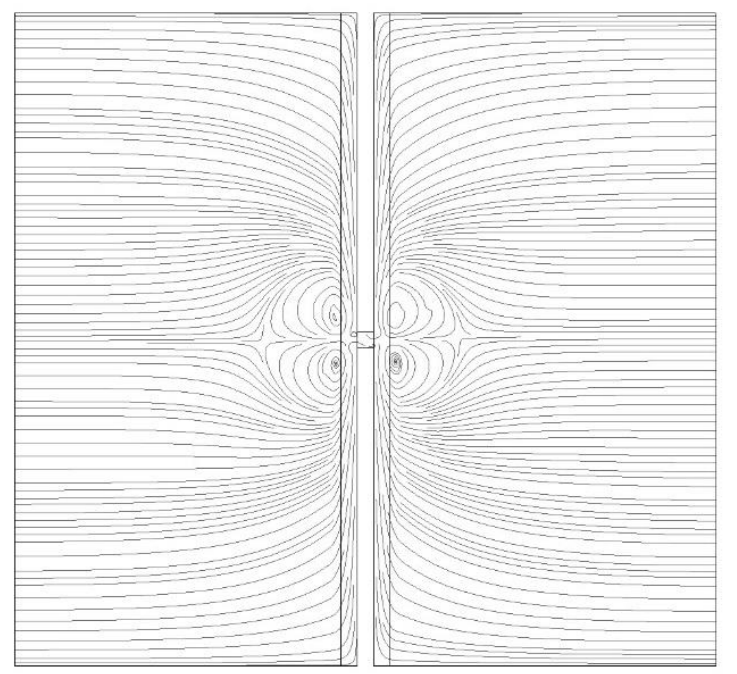

Fig.5. Distribution of spin current in the system with paramagnetic spacer. $l_{s f}{ }^{F}=50 \mathrm{~nm}$.

For comparison of the angular dependence of GMR effect in Levy-Fert and Waintal models we used a reduced magnetoresistance introduced by Slonczewski [11] :

$$
r=\frac{1-\cos ^{2}(\theta / 2)}{1+\chi \cos ^{2}(\theta / 2)}
$$

Where $\chi$ is a parameter directly connected with material properties and which describes the deviation of the $r$ from linearity in respect to $\cos ^{2}(\theta / 2)$. We calculated parameter $\chi$ for different numerical simulation data obtained using Waintal model for different $l_{T}$ and $l_{L}$ values and also for data obtained using Levy-Fert model when $l_{J}=1 \mathrm{~nm}$ (Fig. 6).

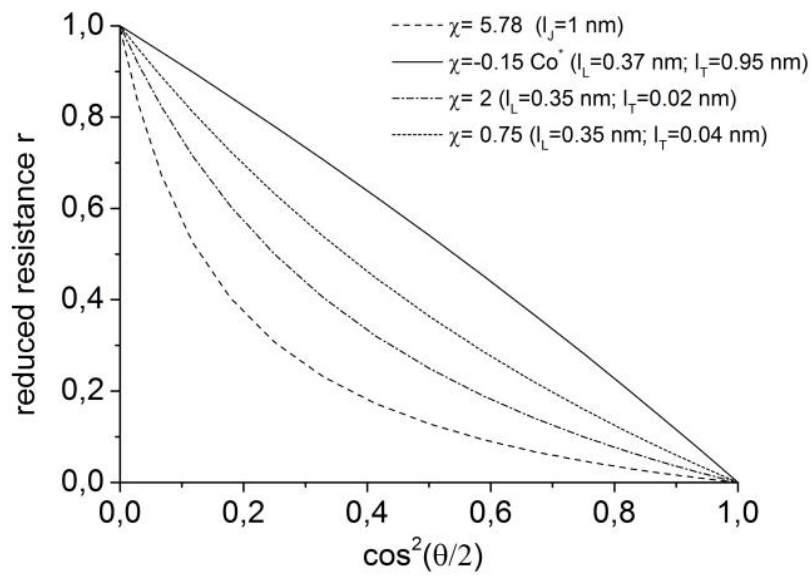

Fig.6. Angular dependence of reduced magnetoresistance for different values of $l_{T}, l_{L}$ and $l_{J}$ obtained from numerical simulation data using Levy-Fert and Waintal models. $l_{s f}{ }^{F}=50$ $\mathrm{nm}$.

For values of $l_{T}=0.95 \mathrm{~nm}$ and $l_{L}=0.37 \mathrm{~nm}$ corresponding to Co taken from Waintal et al the value of $\chi$ parameter equals to -0.15 . However, the experimental data [12] shows that the value of $\chi$ is in the range of 1.2 to 1.6 . The values of calculated $\chi$ close to ones observed in experiment correspond to the curves with $\chi$ equals to 2 and 0.75 . These values are obtained in case if $l_{T}$ has a rather small value. It means that transverse component of spin accumulation is destroyed by strong spin flip scattering on the interface.

\section{Conclusions}

Finite element approach was applied to investigate spin transport properties of structures with complex geometry. It was shown, that application of magnetic pillars is more efficient from the point of GMR effect value than application of a thin paramagnetic spacer. As non-uniformity of spin currents has a significant impact on the GMR effect value, it was shown that application of number of pillars is more effective than application of one pillar of equivalent diameter. Also non-uniform distribution of spin currents leads to occurrence of spin current vortices. Despite the fact that spin transport equations presented by Waintal et al give the same GMR effect as spin transport equations presented by LevyFert, they give a better description of angular dependence of the GMR effect.

\section{References}

1. M. N. Baibich, J. M. Broto, A. Fert, F. N. Van Dau, F. Petroff, P. Etienne, G. Creuzet, A. Friederich, and J. Chazelas, Phys. Rev. Lett. 61, 2472 (1988).

2. G. Binasch, P. Grünberg, F. Saurenbach, and W. Zinn, Phys. Rev. B 39, 4828 (1989). 
3. R. E. Camley and J. Barnaś, Phys. Rev. Lett. 63, 664 (1989).

4. P. M. Levy, S. Zhang, and A. Fert, Phys. Rev. Lett. 65, 1643 (1990).

5. A. Vedyayev, M. Chshiev, N. Ryzhanova, B. Dieny, C. Cowache, and F. Brouers, J. Magn. Magn. Mater.

172, 53 (1997).

6. S. Zhang, P. M. Levy, and A. Fert, Phys. Rev. Lett.

88, (2002).

7. A. Vedyayev, B. Dieny, and N. Ryzhanova, Europhys. Lett. EPL 19, 329 (1992).

8. T. Valet and A. Fert, Phys. Rev. B 48, 7099 (1993).

9. C. Petitjean, D. Luc, and X. Waintal, Phys. Rev. Lett.

109, (2012).

10. N. Strelkov, A. Vedyayev, N. Ryzhanova, D.

Gusakova, L. D. Buda-Prejbeanu, M. Chshiev, S.

Amara, N. de Mestier, C. Baraduc, and B. Dieny, Phys.

Rev. B 84, (2011).

11. J. . Slonczewski, J. Magn. Magn. Mater. 247, 324

(2002).

12. L. Giacomoni, B. Dieny, W. P. Pratt, R. Loloee, and M. Tsoi, (n.d.). 